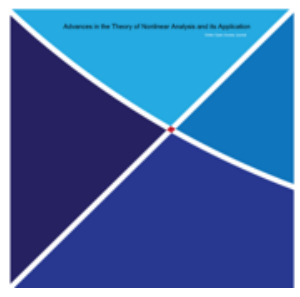

\title{
Modeling of Tumor-Immune System Interaction with Stochastic Hybrid Systems with Memory: A Piecewise Linear Approach
}

\author{
Nurgül Gökgöz ${ }^{a}$, Hakan Öktem ${ }^{b}$ \\ ${ }^{a}$ Department of Mathematics, Çankaya University, Ankara, Turkey. \\ ${ }^{b}$ Department of Aviation Electrical \& Electronics, 19 Mayıs University, Samsun, Turkey.
}

\begin{abstract}
In this work, we benefit from hybrid systems that are advantageous because of their analytical and computational usefulness in the case of inferential modeling. In fact, many biological and physiological systems exhibit historical responses such that the system and its responses depend on the whole history rather than a combination of historical events. In this work, we use and improve hybrid systems with memory (HSM) in the subclass of piecewise linear differential equations. We also include stochastic calculus to our model to exhibit uncertainties and random perturbations clearly, and we call this model stochastic hybrid systems with memory (SHSM). Finally, we choose tumor-immune system data from the literature and show that the model is capable to model history dependent behavior.
\end{abstract}

Keywords: hybrid systems, functional differential equations, pattern memorization, multistationarity, regulatory dynamical systems.

2010 MSC: 37N25, 34C23, 34K13, 34K20, 92C50.

\section{Introduction}

Tumor dynamics exhibit complex interactions such as immune responses. Immune response to tumor growth has been widely investigated in the literature. Through these models, important parameters have been obtained and some predictions have been estimated and some predictions have been performed [30].

Email addresses: ngokgoz@cankaya.edu.tr (Nurgül Gökgöz), hoktem@gmail.com (Hakan Öktem) 
Some other approaches and models can be given as in references [5, 7, 12]. For a more detailed analysis of further models in the literature, one may refer to [1], and the references therein. In the case of potential use in treatment planning, instead of applying first-principles models which require a lot of a priori information which might not be available, we employ a model that has the ability of adapting to a subject by subject variability and to unknown factors; this has the advantage of suggesting the best choice for each case. One of the well-known solution approaches to this problem is, inferring parameters and deciding on the behavior of the system based on empirical observations. In inferential modeling, a class of model is chosen and the parameters are derived from the observations. In such a case, hybrid systems, which can be described as systems formed by continuous and Boolean variables regulating each other [24, are useful in inferential modeling because of their analytical and computational advantages. Hybrid systems also offer several advances for various modeling [14, 16] and theoretical problems [15] in natural sciences. In order to investigate different applications and various modeling versions and analyses of hybrid systems, one may see [6, 19, 32] and the references given there. Moreover, for piecewise linear approaches applied in regulatory systems, [37] offers a good source.

One of the most important properties of a regulatory system is that it is able to memorize its history. In other words, a combination of the previous inputs of the system decides its stationary behavior, and in turn, the system's stationary state decides on the response of the system's future external input. This is the crucial mechanism for adoption and learning in these systems. Delbruck's suggestion mentions that multistationarity, which is the existence of more than one stationary steady state of a system, might be reproduced by epigenetic differentiation in gene networks [10, 42]. Subclasses of hybrid systems, like piecewise linear ones, which are typical examples of non-trivial multistationary systems, are successfully used in modeling gene networks [14]. Some other models of similar phenomena which are also abstractions of hybrid systems, such as Boolean Networks [26, 42] and Boolean Delay Equations (BDE) [9, 35] are given, too. History-dependent responses of perturbations, or future inputs, can be obtained through the co-existence of multistationarity with delays. In [41, it has been stated that time delays could affect the stimulus responses in gene networks and, therefore, the answer of a model to a stimulus is history dependent. Some of such behaviors were demonstrated with the help of BDE [36]. By using hybrid systems with memory phenomena, one can efficiently model the representation of factors which may depend on the whole history, rather than on a combination of historical events. Systems which use existing past information and involve it for intervention or decision making are known to be history dependent: associative memory (neural system) [3] and immune response [23]. Therefore, to consider functional extensions of hybrid systems is a strong motivation. Furthermore, involving delays in the piecewise constant part of hybrid systems was suggested to handle the analytical and computational difficulties of traditional delay differential equations (DDEs) with arbitrary initial functions [25]. Different types of Functional Differential Equations (FDEs), which are generally developed by considering naturally occurring delays in dynamical systems [4], can establish the history-dependent behavior observed in biological systems [8, 38]. For a more general and abstract model of hybrid systems with memory, one may refer to [31]. In this work, we construct a subclass of hybrid systems with memory to use in inferential modeling. In order to realize this goal, we propose to use Stochastic Hybrid System with Memory (SHSM) which differs from Hybrid Systems with Memory (HSM) by their flows and employ this approach to tumor-immune interaction dynamics. To find the parameter values of the model, we benefit from SDE Toolbox in MATLAB. We find parameter values for every state of the model. Here, we benefit from data that can be found the literature [13], in order to obtain more realistic values. The experiment that the authors of the reference [13 have conducted and the data values that they have obtained can be summarized as follows: Essentially, there are two different groups of mice and they are examined under IL1- $\alpha$ effect which is a variable in immune system. The group with IL1- $\alpha$ in their system is capable of defending itself against tumor growth, and the other group fails in this process. Their defense mechanisms and cancer growth processes differ widely. This shows us that the memorization ability of the immune system is crucial for the organism. The biological background can be found in Section 3 . In this work, we consider a piecewise linear appraoch and for a nonlinear version, one may see [22]. 


\section{Mathematical Model and Illustrative Examples}

A typical hybrid system $H=(Q, Y, \operatorname{Init}, f, \operatorname{Inv}, E, G, R)$ is formally defined as a combination of discrete states, continuous variables, initial states, vector fields, invariant sets, edges, guard conditions and reset maps [24, 39]. Some extensions of hybrid systems are hybrid I/O systems which consider external inputs to the system [33], hybrid control systems involving a controller to determine a set of inputs forcing the system to supposedly optimal states [18], stochastic hybrid systems involving random perturbations to the system [29].

In the subsequent representation, a memory set has been included for the purpose of investigation of the behavior's dependence on history. Furthermore, we give the execution of the model. In fact, the behavior of the system and its response to external inputs are determined by the whole history rather than the initial values in many classes of switching systems in nature and technology [20, 41]. Particularly, modeling some biological systems that exhibits history memorization it is necessary to involve an element to keep such information. More precisely, we have the following definition for Hybrid Systems with Memory.

A Hybrid system with memory $H$ is [20, 22];

$$
H=(Q, X, U, T, \text { Init }, M, f, \text { Inv }, E, G, R)
$$

consisting of

- a set of discrete states $Q=\left\{q_{1}, \ldots, q_{m}\right\}$ which are also called locations,

- a space of continuous variables $X=\mathbf{R}^{n}$,

- a set of initial conditions Init $\subseteq Q \times X \times M$,

- a space of inputs $U=\mathbf{R}^{z}$ (control, disturbance or both),

- a space of independent variables $T=\mathbf{R}^{k}$, typically the time $T=\left[t_{0}, \infty\right)$,

- a vector field $f: Q \times X \times U \times M \longrightarrow X$, governing the continuous evolution,

- an invariant set (domain, subspace) for each $q \in Q$, Inv $: Q \longrightarrow P(X)$ where $P(\cdot)$ denotes the power set. Each state's governing dynamics is valid within its invariant set;

- a set of edges (state transitions) $E \subset Q \times Q$,

- guard conditions for each edge $G: E \times M \longrightarrow P(X)$,

- a reset map for each edge $R: E \times X \times U \longrightarrow P(X)$,

- $M(t) \in M$ is a growing memory of past state transitions such that

- $M(0)=\left\{M_{0}\right\}=\left\{\left(t_{0}, x_{0}, q_{0}\right)\right\}$,

- if $M\left(t^{-}\right)=\left\{M_{0}, M_{1}, \ldots, M_{i}\right\}$ and $x\left(t_{j}\right) \in g\{q(t), q \in Q\}$, then $M\left(t^{+}\right)=\left\{M\left(t^{-}\right), M_{i+1}\right\}$,

- $M_{i+1}=\left(t_{j}, x\left(t_{j}^{-}\right), q\left(t_{j}^{-}\right)\right)$

In this definition, the previous progress of the system, containing the values of variables and the time before and after the state transition, is sampled at state transitions. The memory set, $M(t)$, is a piecewise constant variable between state transitions and, moreover, the memory grows at each state transition. Thus, a HSM 
has a complexity that is increasing with time. A subclass of HSM can be considered as a piecewise linear hybrid system with memory, and it can be described with a state space description as follows [20]:

$$
\begin{aligned}
\frac{d x}{d t} & =A_{q(t), M(t)} x(t)+B_{q(t), M(t)} u(t)+k_{q(t), M(t)}, \\
x(0) & =x_{0}, \quad q(0)=q_{0} \\
q(t) & =q_{j} \text { if } x(t) \in X_{j} .
\end{aligned}
$$

If $x\left(t_{0}^{-}\right) \in X_{j}, x\left(t_{0}^{+}\right) \notin X_{j}$ and $M\left(t_{0}^{-}\right)=\left\{M_{1}, \ldots, M_{k}\right\}$, then,

$$
\begin{aligned}
M\left(t_{0}^{+}\right) & =\left\{M\left(t_{0}^{-}\right), M_{k+1}\right\}, \\
M_{k+1} & =\left\{t_{0}, x\left(t_{0}^{-}\right), x\left(t_{0}^{+}\right)\right\} .
\end{aligned}
$$

An example for the stochastic case can be given. Let $Q=\left\{q_{0}, q_{1}, q_{2}\right\}, Y=\mathbb{R}$, Init $=\left\{q=q_{0}, y_{0} \in\left(b_{1}, b_{2}\right)\right\}$, $\operatorname{Inv}\left(q_{0}\right)=y_{0} \in\left(b_{1}, b_{2}\right)$. The system evolution at the initial state and until it hits one of the boundaries of the initial set $b_{1}$ or $b_{2}$, looks as follows:

$$
\text { if } \quad q=q_{0} \text { then, } \quad d y_{t}=\sigma_{0} Y_{t} d W_{t},
$$

The above stochastic system is Geometric Brownian Motion without drift; hence its solution can be found widely in the literature. We give the solution under Itô interpretation:

$$
y_{t}=y_{0} \exp \left\{\sigma_{0} W_{t}-\frac{1}{2} \sigma^{2} t\right\} .
$$

The hitting times of each barrier are defined by stopping times as seen in the literature:

$$
\begin{aligned}
\tau_{1} & =\inf \left\{t \in \mathbb{R}^{+}: Y_{t} \geq b_{2}\right\}, \\
\tau_{2} & =\inf \left\{t \in \mathbb{R}^{+}: Y_{t} \leq b_{1}\right\}
\end{aligned}
$$

Here, $\tau_{1}$ and $\tau_{2}$ are random variables. Thus, in each run, and when the first transition occurs by hitting $b_{1}$ or $b_{2}$ is totally random. Thus, as a first transition time we take $\tau^{*}=\min \left\{\tau_{1}, \tau_{2}\right\}$ and so the memory set will be

$$
M=\left\{m_{0},\left(\tau^{*}, b\right)\right\}=\left\{\begin{array}{lll}
\left\{m_{0},\left(\tau^{*}, b_{1}\right)\right\}, & \text { if } \quad y_{\tau^{*}}=b_{1}, \\
\left\{m_{0},\left(\tau^{*}, b_{2}\right)\right\}, & \text { if } \quad y_{\tau^{*}}=b_{2} .
\end{array}\right.
$$

Just as the system hits one of the boundaries, it experiences a differentiation depending on the barrier that got hit.

$$
\begin{array}{r}
q(t)=\left\{\begin{array}{lll}
q_{1}, & \text { if } & b=b_{1}, \\
q_{2}, & \text { if } & b=b_{2},
\end{array}\right. \\
d Y_{t}=\left\{\begin{array}{lll}
-a_{1}\left[Y_{t}-c_{1}\right] d t+\sigma_{1} d W_{t}, & \text { if } & q(t)=q_{1}, \\
-a_{2}\left[Y_{t}-c_{2}\right] d t+\sigma_{2} d W_{t}, & \text { if } & q(t)=q_{2} .
\end{array}\right.
\end{array}
$$

Thus, our system can exhibit two different behaviors and, moreover, it has two different asymptotic distributions depending on its memory set. The stochastic systems used during each state are known as Ornstein-Uhlenbeck processes and have been widely used because of their mean reversion properties. Moreover, they have a stationary probability distribution unlike Brownian Motions. Thus, our system can develop according to two different stationary probability distributions and revert to two different means. The model solution is given by

$$
Y_{t}=\left\{\begin{array}{lll}
y_{0} e^{-a_{1} t}+c_{1}\left(1-e^{-a_{1} t}\right)+\int_{0}^{t} \sigma_{1} e^{a_{1}(s-t)} d W_{s}, & \text { if } & q(t)=q_{1}, \\
y_{0} e^{-a_{2} t}+c_{2}\left(1-e^{-a_{2} t}\right)+\int_{0}^{t} \sigma_{2} e^{a_{2}(s-t)} d W_{s}, & \text { if } & q(t)=q_{2},
\end{array}\right.
$$


where $y_{0}$ is assumed as constant from the initial set. Thus, the distribution of the system after differentiation is apparently a Gaussian distribution with possible two distinct means and variance values. The system's behavior will be

$$
\begin{array}{r}
\mathbb{E}\left(Y_{t}\right)=y_{0} e^{-a_{i} t}+c_{i}\left(1-e^{-a_{i} t}\right), \\
\mathbb{V}\left(Y_{t}\right)=\frac{\sigma_{i}^{2}}{2 a_{i}}\left(1-e^{-2 a_{i} t}\right),
\end{array}
$$

where $i=1$ if $b_{1}$ is achieved first, and $i=2$ otherwise.

\section{Biological Background and Application on Tumor-Immune System Dynamics}

IL-1 $\alpha$ inhibitors are being produced to avoid inflammation, and the advancement of fever and sepsis; by this way, they treat diseases. In the regulation of the immune responses, they play an important role. For detailed information on IL-1 $\alpha$, one may refer to [11, 13, 34] and closer references provided there.

In [13], this effect of IL- $1 \alpha$ inhibitors has been examined through some experiments, applied on different groups of mice. In a comparative study where the authors of [13] have investigated spleen cells from mice injected with an IL1- $\alpha$-positive (Clone 2) cell line or a non-IL1- $\alpha$-expressing (Clone 5) fibrosarcoma cell line, they have analyzed tumor-immune responses. All IL1- $\alpha$-positive fibrosarcoma clones induced regression of tumor growth when they have been injected into mice. At first stages of the experiment, cells started to multiply and accordingly regressed (see Figure 1). On the other hand, cells of non-IL1- $\alpha$-expressing clones grow in a progressive manner and this results in the death of mice [13. Both classes of cell lines (IL1- $\alpha$ positive and -negative) exhibit almost identical growth progress, initially. Their work expresses the role of tumor cell-associated IL1- $\alpha$ in the induction of particular immune responses, ultimately leading to tumor regression and the improvement of an immune memory, which saves the mice from a battle with the violent tumor cells [13].

In our modeling framework, we will use the data of the experiment conducted by Dvorkin et al. [13]. We involve those data values which show tumor diameters of Clone 2 and Clone 5 according to days; in the setting of experiment Clone 2 has been injected with IL1- $\alpha$, whereas Clone 5 has not been. As we have mentioned formerly, different levels of tumor growth and effector cells have been observed, depending on different values of IL1- $\alpha$. Figure 1, Figure 2 and Figure 3 represent these effects graphically. Furthermore, the exact values may be observed from the Table 1. There, S.I. value refers to the Stimulator Index which corresponds to the ratio for immune cells (the effector cell and stimulator cells) and the tumor size has been measured in millimeters $(\mathrm{mm})$ in that table. By examining the data values on the table, one can see that Clone 2 and Clone 5 behave similarly until day 3 and after day 3, Stimulation Index decreases in Clone 5, and after day 15 the tumor size is increasing in Clone 5. In order to illustrate those values in our model, we have designed a system in such a way that we partition it into 4 main states: first state, i.e., $q_{1}$, shows the behavior of both Clone 2 and Clone 5 until day 3; second state, i.e., $q_{m}, m=11,12$, include slightly different behaviors of Clone 2 and Clone 5 until day 15; third and fourth states, i.e., $q_{2}$ and $q_{3}$, respectively, show entirely different actions on tumor growth and S.I. values.

For SHSM simulation purposes, we have prefered Ornstein-Uhlenbeck type of stochastic differential equations and splitted up the system due to the data values with the following manner:

$$
\begin{aligned}
d X^{1}(t) & =\left[\beta_{11}^{q_{k}, m_{i}} \alpha_{1}^{q_{k}, m_{i}}+\beta_{12}^{q_{k}, m_{i}} \alpha_{2}^{q_{k}, m_{i}}-\beta_{11}^{q_{k}, m_{i}} X_{t}^{1}-\beta_{12}^{q_{k}, m_{i}} X_{t}^{2}\right] d t+\sigma_{1}^{q_{k}, m_{i}} d W_{t}^{1}, \\
d X^{2}(t) & =\left[\beta_{21}^{q_{k}, m_{i}} \alpha_{2}^{q_{k}, m_{i}}+\beta_{22}^{q_{k}, m_{i}} \alpha_{2}^{q_{k}, m_{i}}-\beta_{21}^{q_{k}, m_{i}} X_{t}^{1}-\beta_{22}^{q_{k}, m_{i}} X_{t}^{2}\right] d t+\sigma_{1}^{q_{k}, m_{i}} d W_{t}^{2} .
\end{aligned}
$$

Depending on various memory values and different states, we have different parameter values: $\beta_{11}^{q_{k}, m_{i}}$, $\beta_{12}^{q_{k}, m_{i}}, \beta_{21}^{q_{k}, m_{i}}, \beta_{22}^{q_{k}, m_{i}}, \alpha_{1}^{q_{k}, m_{i}}, \alpha_{2}^{q_{k}, m_{i}}, \sigma_{1}^{q_{k}, m_{i}}, \sigma_{2}^{q_{k}, m_{i}}$ where $q_{k} \in\left\{q_{1}, q_{11}, q_{12}, q_{2}, q_{3}\right\}$ and $i=1,2, \ldots$ Network representation of the system can be seen in Figure 4 . Now, let us consider a linear SDE:

$$
d X_{t}=\alpha\left(t, X_{t}\right) d t+\beta\left(t, X_{t}\right) d W_{t},
$$

where

$$
\alpha\left(t, X_{t}\right)=a_{1}(t) X_{t}+a_{2}(t)
$$




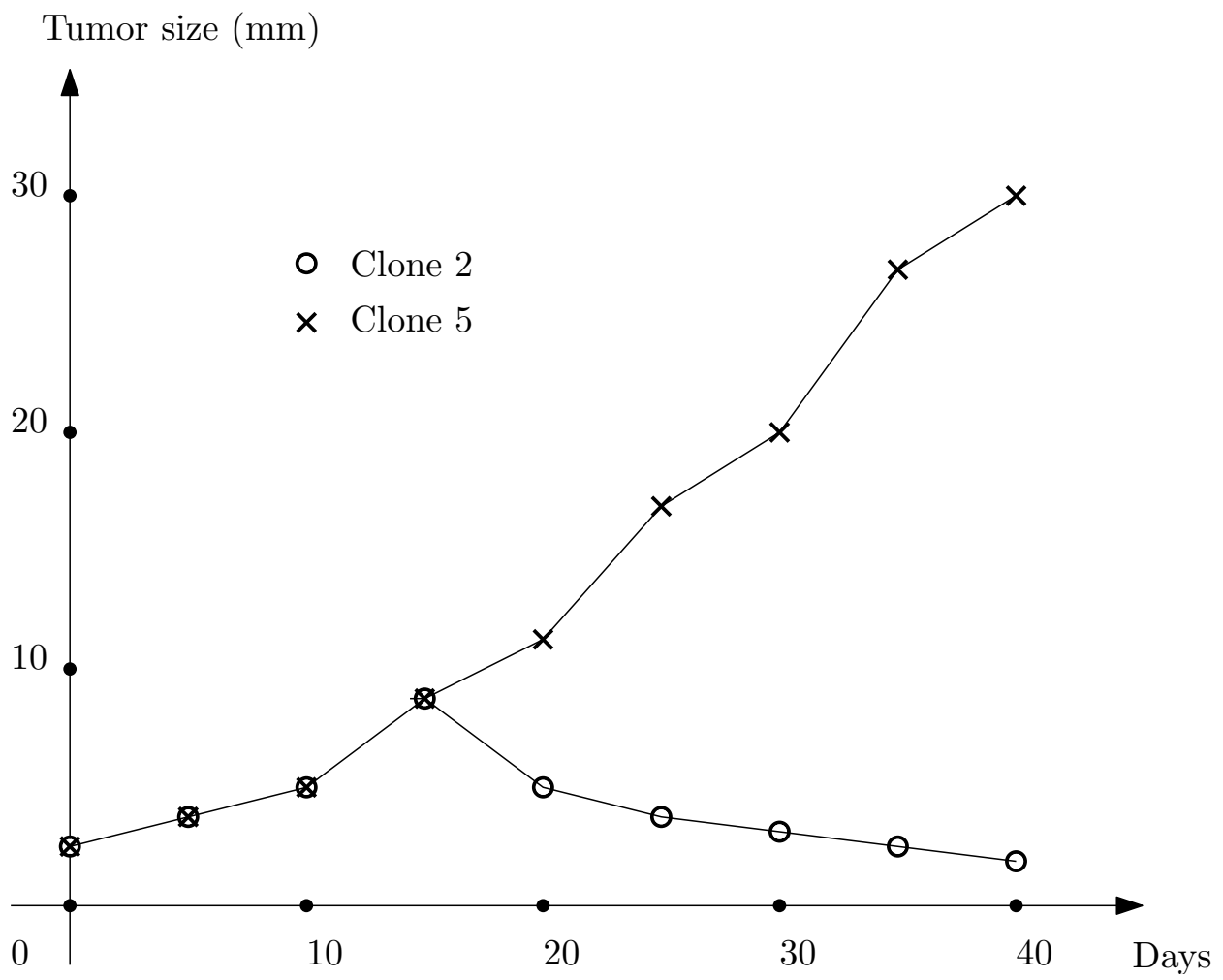

Figure 1: Clone 2 and Clone 5 tumor growth (in $\mathrm{mm}$ ) depending on days [13, 21].

\begin{tabular}{|l||l|l||l|l|}
\hline \multicolumn{1}{|c||}{} & \multicolumn{2}{l|}{ Clone 5} & \multicolumn{2}{l|}{ Clone 2} \\
\cline { 2 - 5 } Days & S.I. & Size of Tumor $(\mathrm{mm})$ & S.I. & Size of Tumor $(\mathrm{mm})$ \\
\hline \hline 0 & 1 & 3.125 & 1 & 3.05 \\
3 & 2.129 & 3.75 & 1.988 & 3.7 \\
7 & 1.443 & 4 & 2.344 & 4.35 \\
10 & 0.914 & 5.5 & 2.822 & 6.35 \\
15 & 0.914 & 8.5 & 3.011 & 7.345 \\
20 & 0.886 & 15.125 & 3.411 & 6 \\
40 & 0.943 & 29.125 & 3.266 & 3.7 \\
\hline
\end{tabular}

Table 1: Stimulator Index, S.I., and tumor growth values for Clone 5 and Clone 2 [21].

$$
\beta\left(t, X_{t}\right)=b_{1}(t) X_{t}+b_{2}(t) .
$$

For a general piecewise linear model, we use a similar statement in the works of Gebert et al. [17] and Öktem [37], with a version which includes the memory variables;

$$
\begin{aligned}
& \alpha\left(t+1, X_{t+1}\right)=M_{1}^{q_{k}, m_{i}} X_{t}+k_{1}^{q_{k}, m_{i}}(t), \\
& \beta\left(t+1, X_{t+1}\right)=M_{2}^{q_{k}, m_{i}} X_{t}+k_{2}^{q_{k}, m_{i}}(t),
\end{aligned}
$$

where $M_{1}^{s(t)}$ and $M_{2}^{s(t)}$ are matrices and $k_{1}$ and $k_{2}$ are vectors. Then the linear SDE can be represented by

$$
d X_{t+1}=\left(M_{1}^{q_{k}, m_{i}} X_{t}+k_{1}^{q_{k}, m_{i}}(t)\right) X_{t}+\left(M_{2}^{q_{k}, m_{i}} X_{t}+k_{2}^{q_{k}, m_{i}}(t)\right) d W_{t}
$$

In this formulation, parameter values are thresholds and focal points. To determine these parameter values, one may refer to [37, 40]. By exchanging equations into a two dimensional Ornstein-Uhlenberg process we get: 


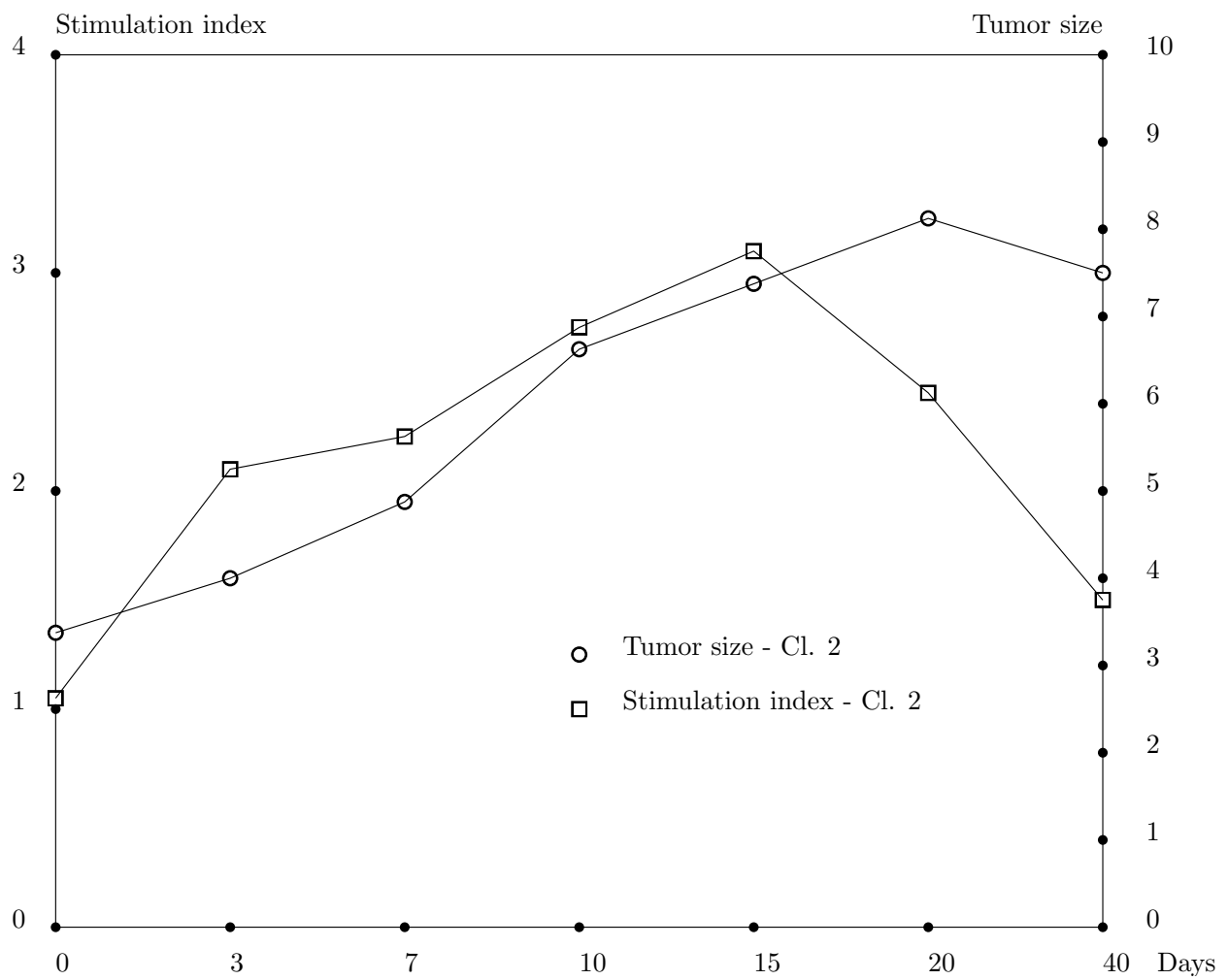

Figure 2: S.I. and tumor growth of Clone 2 depending on days [13, 21].

$$
\left[\begin{array}{l}
d X_{1} \\
d X_{2}
\end{array}\right]=\left[\left[\begin{array}{ll}
\beta_{11}^{q_{k}, m_{i}} & \beta_{12}^{q_{k}, m_{i}} \\
\beta_{21}^{q_{k}}, m_{i} & \beta_{22}^{q_{k}, m_{i}}
\end{array}\right]\left[\begin{array}{c}
\alpha_{1}^{q_{k}, m_{i}} \\
\alpha_{2}^{q_{k}, m_{i}}
\end{array}\right]-\left[\begin{array}{cc}
\beta_{11}^{q_{k}, m_{i}} & \beta_{12}^{q_{k}, m_{i}} \\
\beta_{21}^{q_{k}, m_{i}} & \beta_{22}^{q_{k}, m_{i}}
\end{array}\right]\left[\begin{array}{c}
X_{1} \\
X_{2}
\end{array}\right]\right] d t+\left[\begin{array}{c}
\sigma_{1} d W_{1} \\
\sigma_{2} d W_{2}
\end{array}\right]
$$

or we can write in compact notation:

$$
d X=\left(N^{q_{k}, m_{i}}-M^{q_{k}, m_{i}} X\right) d t+\sigma d W .
$$

In the equation 11, the term $N^{q_{k}, m_{i}}$ is a focal point vector and $M^{q_{k}, m_{i}}$ is a matrix. The memory set contains the first transition in the first state, time and state conditions. It will include and act according to $q_{11}$ or $q_{12}$ :

$$
m=\left\{t(i),\left(\left(X_{1}<2.344 \wedge X_{2}<4\right) \vee\left(X_{1} \geq 2.344 \wedge X_{2} \geq 4\right)\right), q_{m}\right\},
$$

where $i=1,2$. The described method below, that explains how to calculate the expected time for a stochastic process to reach a state or if a stochastic process can reach a state or not, can be found in [2, Chapter8]. By using the same process, we can calculate the time or the state of SHSM. In our model, we can find those values compartmentally. For a stochastic process to reach states $A$ or $B$, can be given by the following procedure. Suppose that $X(t)$ be a stochastic process, and a solution of the stochastic differential equation given by

$$
d X(t)=a(X(t)) d t+b(X(t)) d W(t), \quad X(0)=x,
$$

then the transition probability density function for the linear stochastic process gives us a solution to the backward Kolmogorov differential equation [2, Chapter 8]:

$$
\frac{\partial p(y, x, t)}{\partial t}=a(x) \frac{\partial p(y, x, t)}{\partial x}+\frac{b^{2}(x)}{2} \frac{\partial^{2} p(y, x, t)}{\partial x^{2}} .
$$




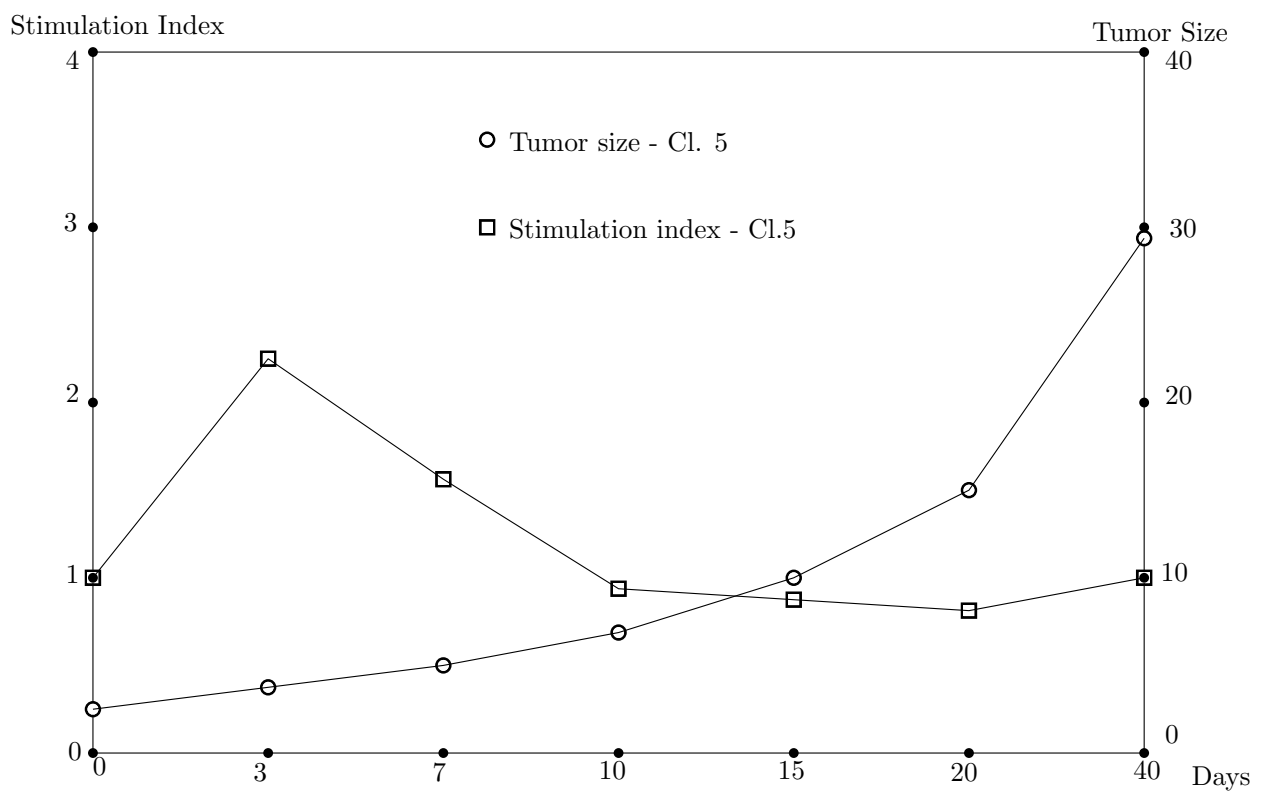

Figure 3: S.I. and tumor growth of Clone 5 depending on days [13 21].

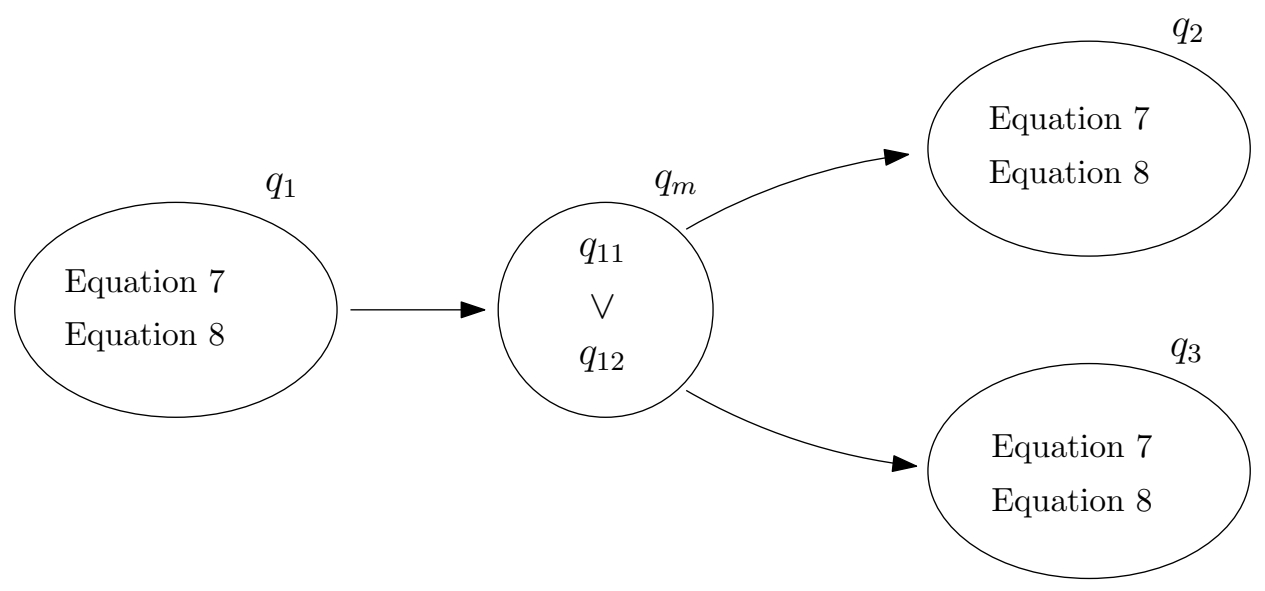

Figure 4: Representation of the states, [21.

If $Q(x, t)$ is the probability that measures whether the process does not reach states $A$ or $B$ in time $[0, t]$, and if $A<x<B$, then we have [2, Chapter 8]:

$$
Q(x, t)=\int_{A}^{B} p(y, x, t) d y .
$$

If $T(x)$ is the random variable which refers the time for the stochastic process to reach states $A$ or $B$, then expected time can be found by the following integral [2, Chapter 8]:

$$
E(T(x))=\int_{0}^{\infty} Q(x, t) d t .
$$

In order to simulate the stochastic dynamics, and estimate parameter values, we have benefited from SDE toolbox in MATLAB. Besides the parameter values, we are able to find Monte-Carlo statistics results including process mean, process variance, process median, confidence interval for the trajectories, process skewness, process kurtosis, process moments by utilizing this toolbox (cf. Figures 5-9). Since we can find the values of the unknown parameters, the equations for each state can also be determined. Some data values have been kept and modeled according to these data in order to test the model. You may see the Figures 10 and 11. 

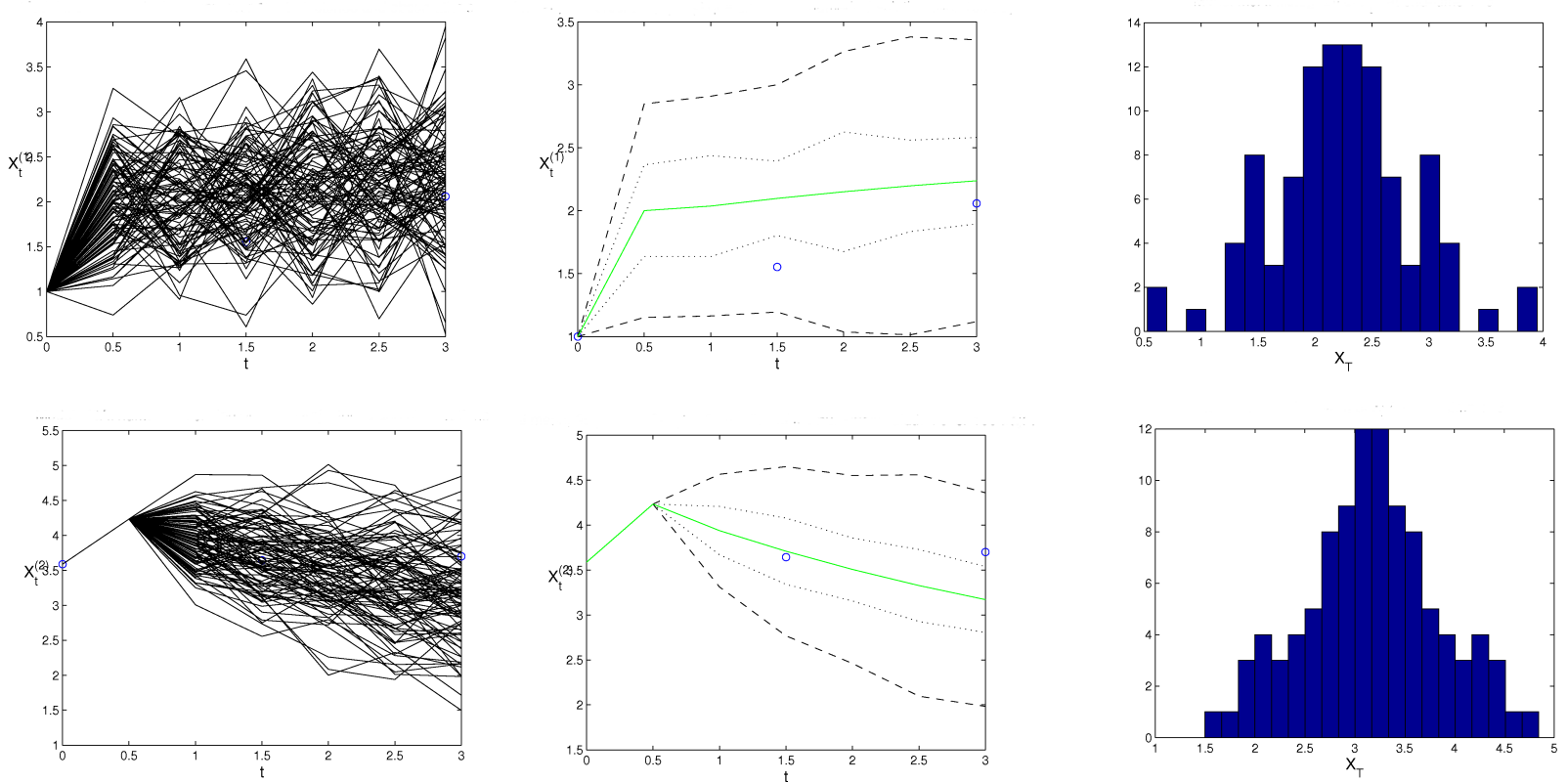

Figure 5: Results of the simulation of Ornstein-Uhlenberg process for state $q_{1}$. On the first row, statistical results for $X_{1}$ and on the second row, the same results for $X_{2}[21$.
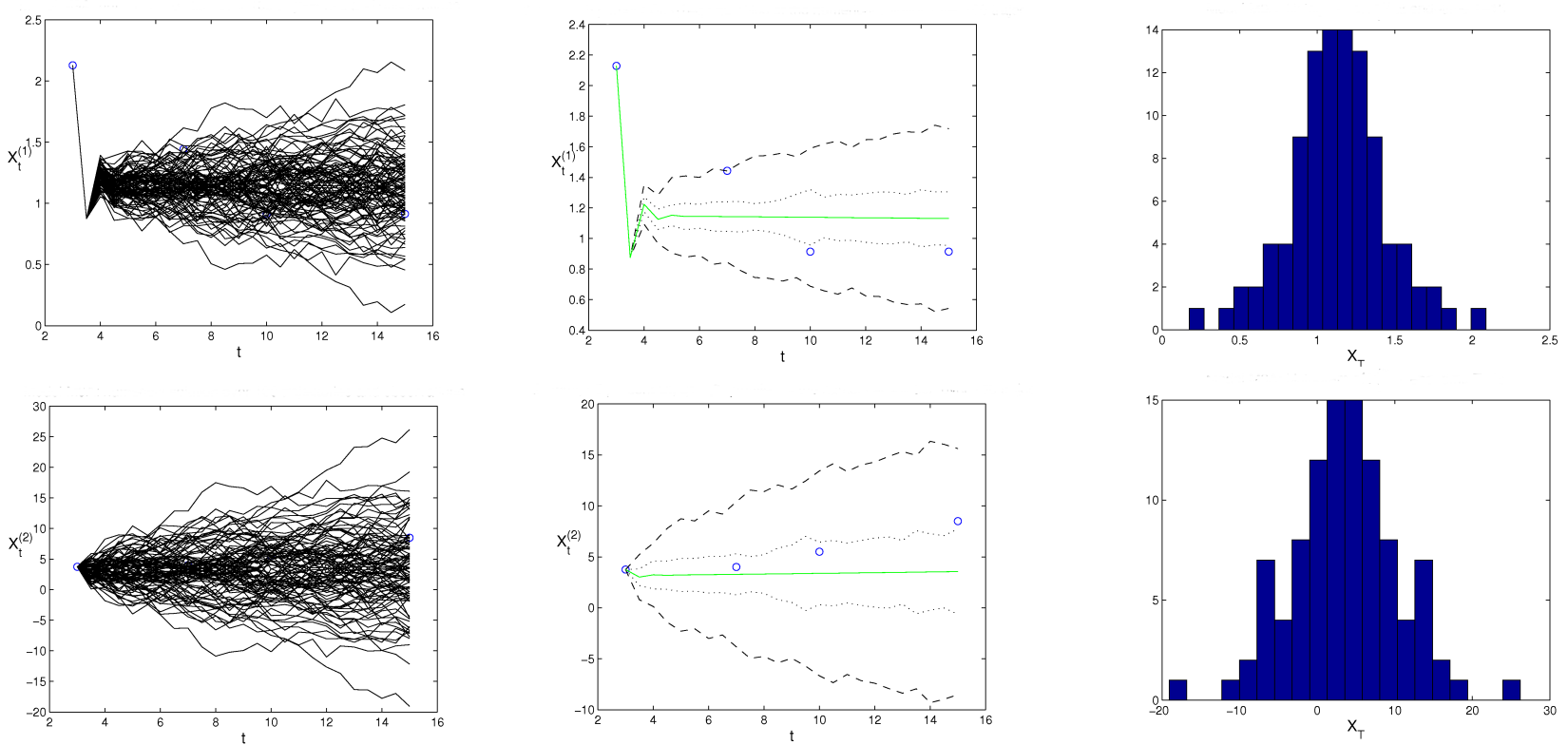

Figure 6: Results of the simulation of Ornstein-Uhlenberg process for state $q_{11}$. On the first row, statistical results for $X_{1}$ and on the second row, the same results for $X_{2}$ [21]. 

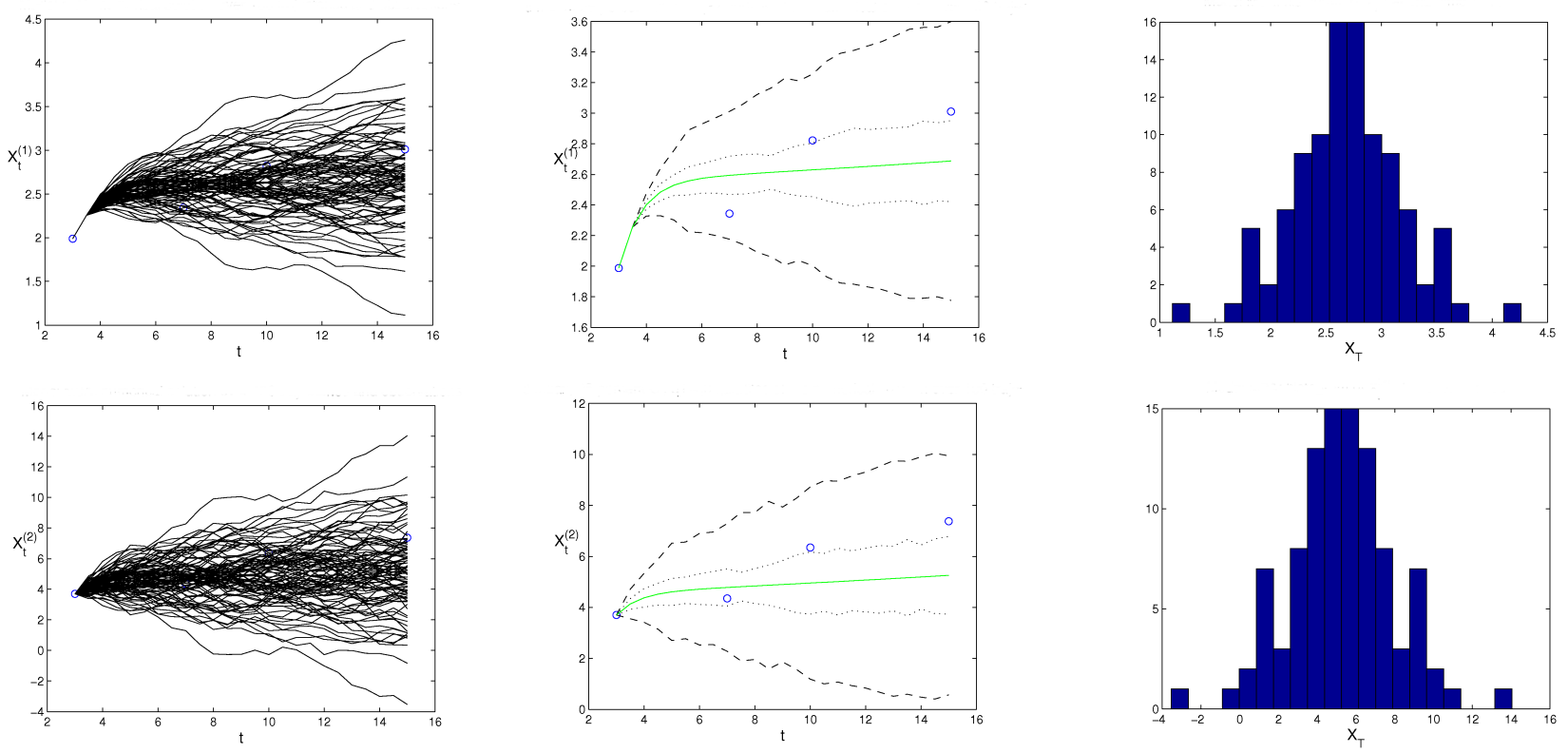

Figure 7: Results of the simulation of Ornstein-Uhlenberg process for state $q_{12}$. On the first row, statistical results for $X_{1}$ and on the second row, the same results for $X_{2}[21$.
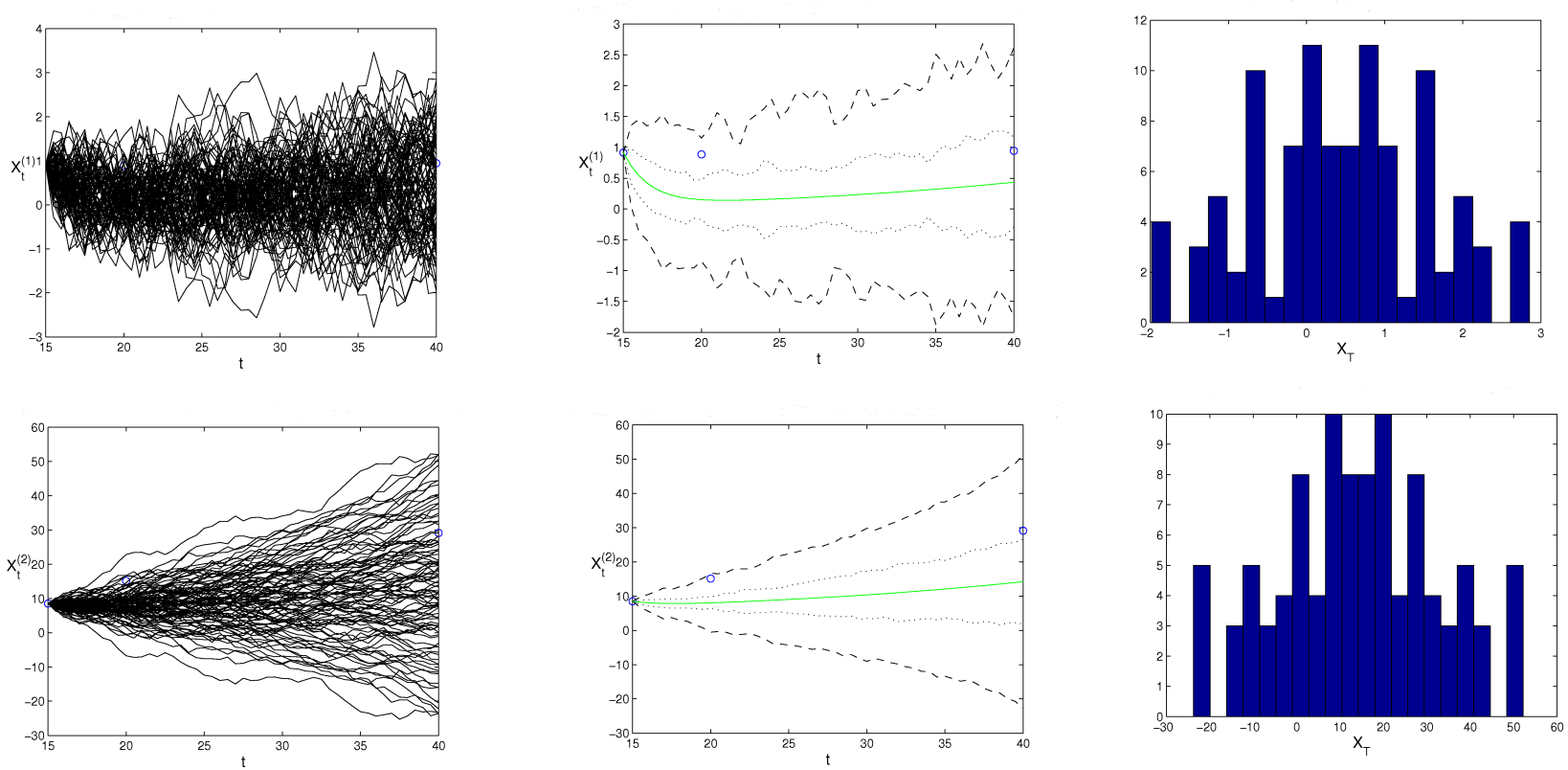

Figure 8: Results of the simulation of Ornstein-Uhlenberg process for state $q_{2}$. On the first row, statistical results for $X_{1}$ and on the second row, the same results for $X_{2}[21]$. 

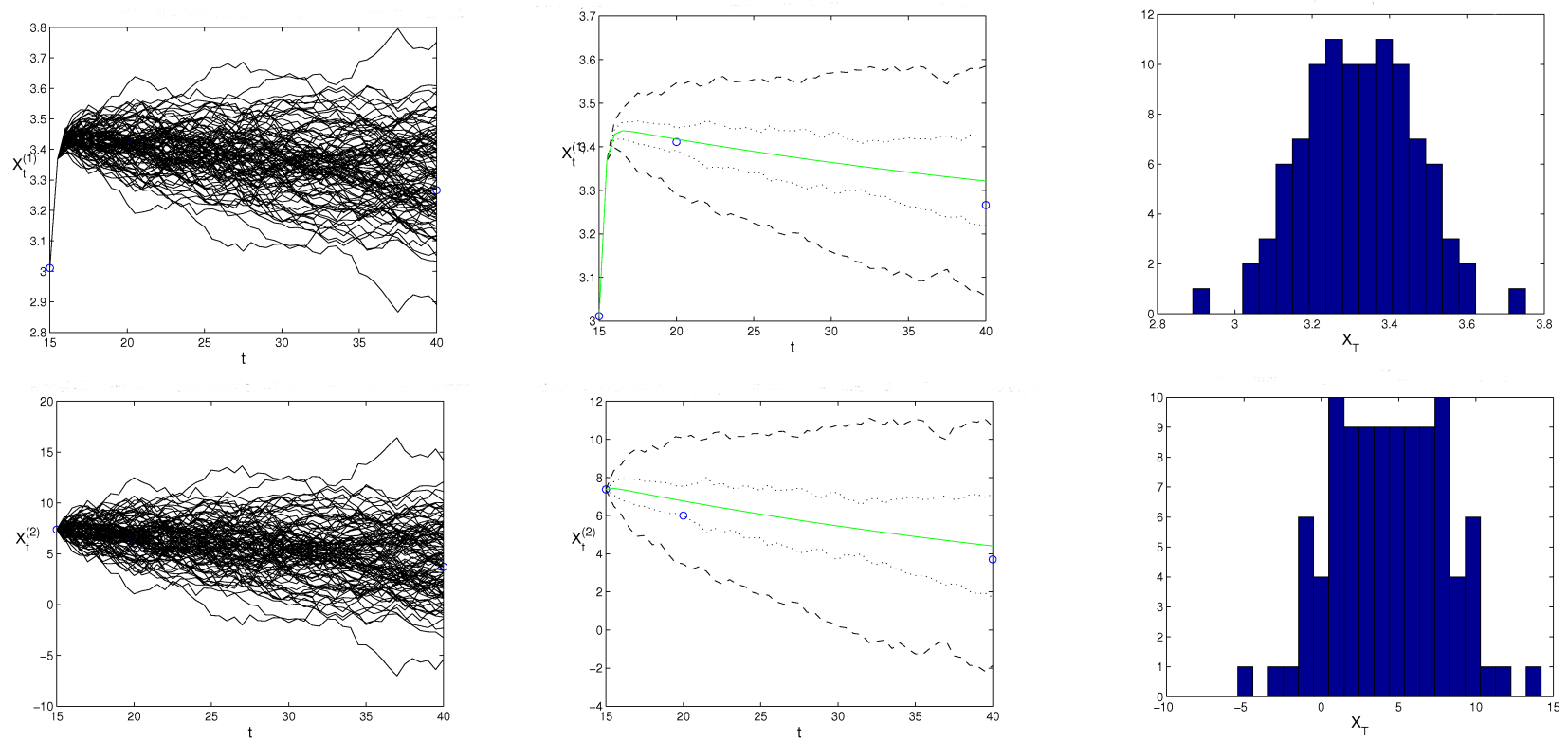

Figure 9: Results of the simulation of Ornstein-Uhlenberg process for state $q_{3}$. On the first row, statistical results for $X_{1}$ and on the second row, the same results for $X_{2}[21$.
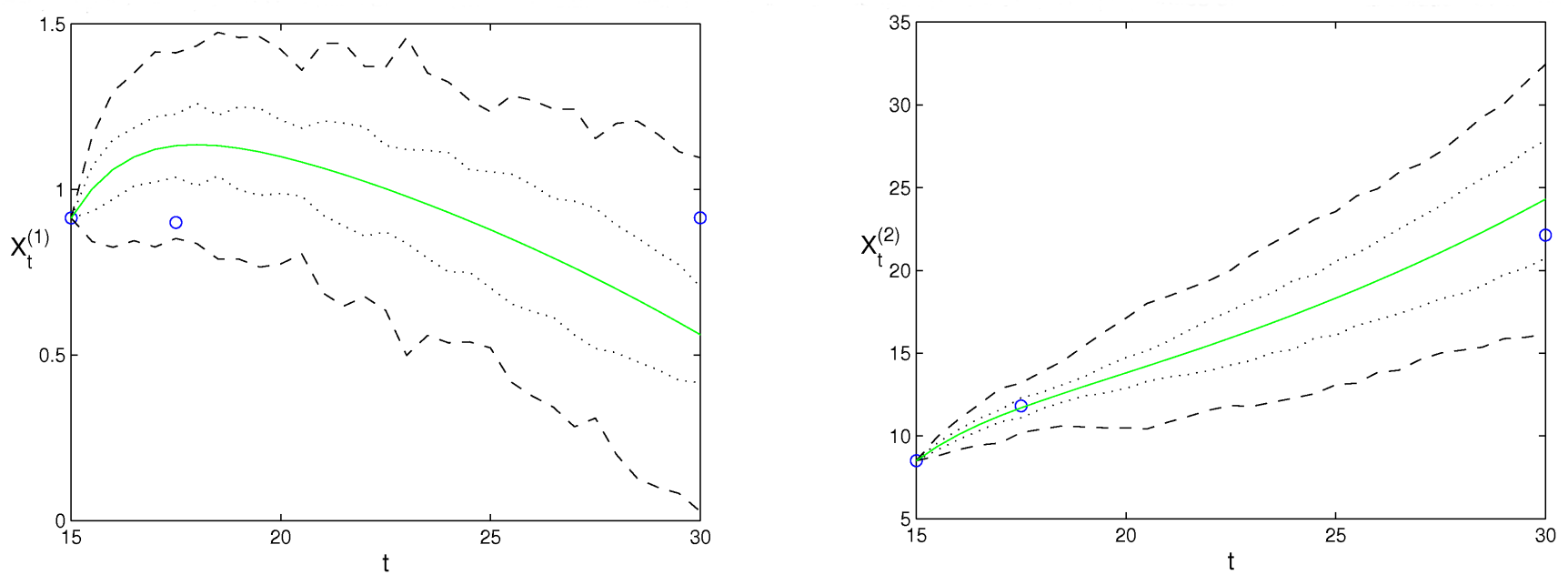

Figure 10: Estimation values for state $q_{2}$. In that state, we expect the system to show a similar behavior as Clone 5. Variable $X_{1}$ (left) and Variable $X_{2}$ (right) [21]. 

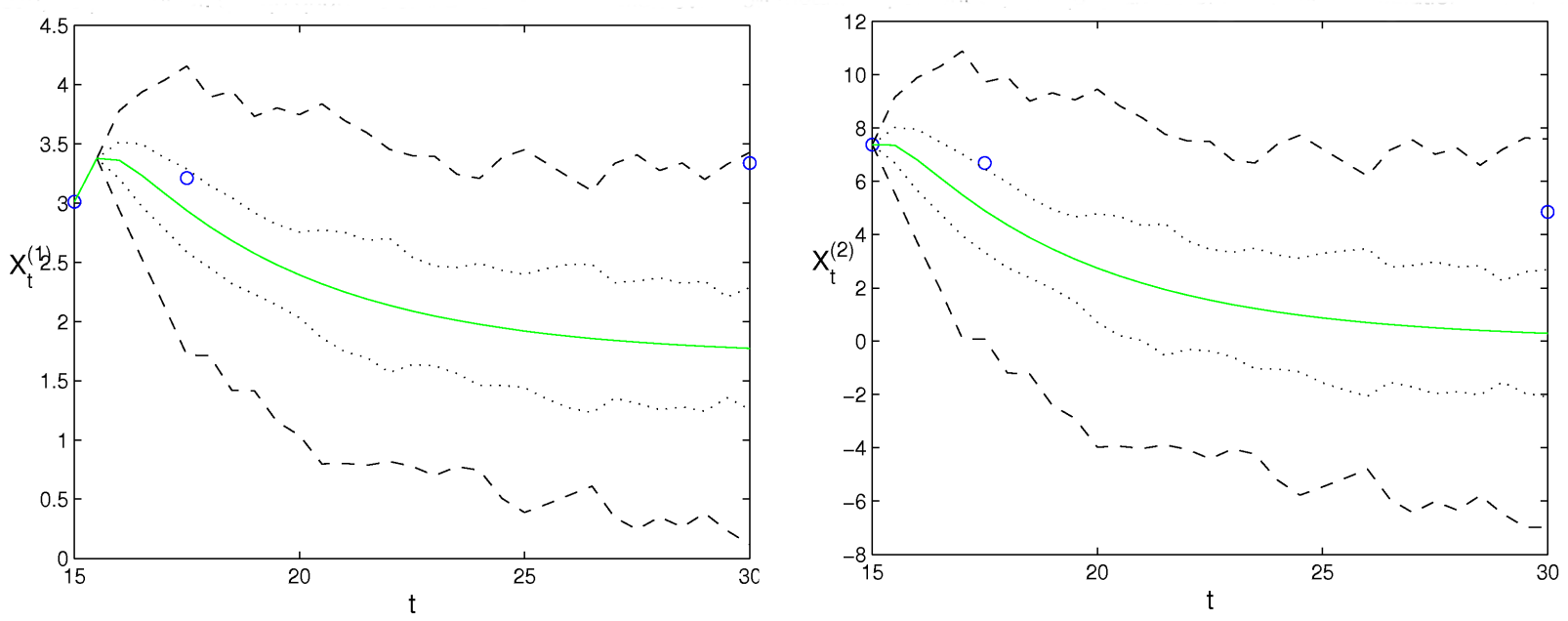

Figure 11: Estimation values for state $q_{3}$. In that state, we expect the system to show a similar behavior as Clone 2 . The variable $X_{1}$ (left) and the variable $X_{2}$ (right) [21].

\section{Discussion}

The biological example used here can be thought of, e.g., as an example to an alternative way to use FDEs or DDEs. In the first state, Clone 2 and Clone 5 acts similarly, and after day 3 , the tumor size starts to change slightly in different clones. Clone 2 remembers the values of $t, q_{1}, \quad x$ and acts between days 3-15 as it did in the past. Therefore, $q_{m}$, which includes $q_{11}$ and $q_{12}$ and the host choosing one of the states according to its memory set, can be considered as the delay state for Clone 2 in the experiment. After day 15 it changes its behavior. This behavior can be considered in such a way that the immune system remembers the memory set of $t, \quad q, \quad x$ and after day 15 it changes its behavior according to IL1- $\alpha$ which can be considered as the control input $u$ of the host. The simulations have been done compartmentally.

\section{Conclusions}

In this work, we utilized Stochastic Hybrid Systems with Memory (SHSM) to model the history dependent behavior in hybrid systems and stochastic hybrid systems. The work can be considered either as introducing memory into stochastic hybrid systems or introducing switching behavior into FDEs. The introduced class can be used in modeling of many systems with pattern memorization capability and subject to external inputs. Various hybrid systems involving delays have already been investigated. We selected a system exhibiting the same pattern, which is simple but not easy to model with traditional hybrid systems. Various types of functional differential equations were also implied in modeling history dependent systems by other scientists. We discussed stochastic case, where stochastic hybrid systems with memory can be a tool for modeling non-Markovian Stochastic Dynamical Systems. In this work, we have seen that our modeling scheme can be employed to model tumor-immune dynamics. We have worked with raw data found from the literature and have seen that complex networks, which exhibits history-dependent behavior, can be modeled in a simpler way by applying this modeling framework where the dynamics of the system is determined by the location of the state vector and the memory. For instance, gene-regulatory networks that has memorization capability can be mimicked by this approach. As a future work, SHSM can be employed in modeling some complicated genetic or neurological systems. Another possible use can be the design of learning adaptive systems or controllers. Moreover, since fractional differential equations are said to capture and exhibit memory dependent behavior, they are used in modeling real world applications [27] and they are used to check stability where the equation is very sensitive to delays [28]. Therefore, an extension of this method to 
fractional differential equations and checking and comparing the stability of the system can also be considered as a future work.

\section{Acknowledgment}

This work is a part of the corresponding author's $\mathrm{PhD}$. Thesis.

\section{Funding}

This work has been supported by The Scientific and Technological Research Council of Turkey (TÜBİTAK) project 104T133.

\section{References}

[1] J.A. Adam, N. Bellomo, A survey of Models for Tumor-Immune System Dynamics, Birkhäuser, Boston, MA, 1996.

[2] L.J.S. Allen, An introduction to stochastic processes with applications to biology. Second edition. CRC Press, Boca Raton, FL, 2011.

[3] U. Bastolla, G. Parisi, Attractors in fully asymmetric neural networks, J. Phys. A: Math. Gen., 30, 5613-5631, 1997.

[4] G.A. Bocharov, F.A. Rihan, Numerical modeling in biosciences using delay differential equations, Journal of Computational and Applied Mathematics, 125, 183-199, 2000.

[5] N. Bellomo, Modeling the hiding-learning dynamics in large living systems, Appl. Math. Lett., 23, 907-911, 2010.

[6] C.G. Cassandras, J. Lygeros, Stochastic Hybrid Systems, CRC Press, FL, 2006.

[7] C. Cattani, A. Ciancio, Hybrid two scales mathematical tools for active particles modeling complex systems with learning hiding dynamics, Mathematical Models and Methods in Applied Sciences Volume 17, Issue 2, Pages 171-187, February 2007.

[8] L. Chen, Stability of Genetic Regulatory Networks With Time Delay, IEEE Transactions on Circuits and Systems I: Fundemental Theory and Applications, Vol. 49, No. 5, May 2002.

[9] D. Dee, M. Ghil, Boolean difference equations, i: Formulation and Dynamic behavior, SIAM J. Appl. Math., 44:111-126, 1984.

[10] M. Delbruck. Discussion, In Unites biologiques douees de continuite genetique, Editions du Centre National de la Recherche Scientifique, Paris. pp. 33-35, 1949.

[11] C.A. Dinarello, Biologic basis for interleukin-1 in disease. Blood, 87, 2095-2147, 1996.

[12] A. D'Onofrio, Metamodeling tumor-immune system interaction, tumor evasion and immunotherapy. Mathematical and Computer Modelling Volume 47, Issues 5-6, Pages 614-637, March 2008.

[13] T. Dvorkin, X. Song, S. Argov, R. M. White, M. Zoller, S. Segal, C. A. Dinarello, E. Voronov, R. N. Apte, Immune phenomena involved in the in vivo regression of fibrosarcoma cells expressing cell-associated IL-1alpha, J Leukoc Biol.; 80(1):96-106, 2006.

[14] R. Edwards, L. Glass, Combinatorial explosion in model gene networks, Journal of Chaos, volume 10, number 3, pp. 691-704, September 2000.

[15] R. Edwards, H.T. Siegelmann, K. Aziza, L. Glass, Symbolic dynamics and computation in model gene networks, Journal of Chaos, volume 11, number 1, pp. 160-169, March 2001.

[16] J. Gebert, H. Öktem, S.W. Pickl, N. Radde, G.-W. Weber, F.B. Yilmaz, Inference of Gene Expression Patterns by Using a Hybrid System Formulation An Algorithmic Approach to Local State Transition Matrices, Anticipative \& Predictive Models in Systems Science 1, pp. 63-66, 2004.

[17] J. Gebert, N. Radde, G. W. Weber. Modeling gene regulatory networks with piecewise linear differential equations, European Journal of Operational Research 181, 1148-1165, 2007.

[18] D. Godbole, J. Lygeros, and S. Sastry, Hierarchical hybrid control: A case study, Lecture Notes in Computer Science, vol. 999, pp. 166-190, 1995.

[19] R. Goebel, R. G. Sanfelice, A. R. Teel, Hybrid Dynamical Systems: Modeling, Stability, and Robustness. Princeton University Press, 2012.

[20] N. Gökgöz. Development of Tools For Modeling Hybrid Systems With Memory, Msc. Thesis, Scientific Computing, Institute of Applied Mathematics, Middle East Technical University, 2008.

[21] N. Gökgöz, Modeling Stochastic Hybrid Systems With Memory With an Application to Immune Response of Cancer Dynamics, PhD Thesis, Scientific Computing, Institute of Applied Mathematics, Middle East Technical University, 2014.

[22] N. Gökgöz , H. Öktem, G. Weber, Modeling of Tumor-Immune Nonlinear Stochastic Dynamics with Hybrid Systems with Memory Approach, Results in Nonlinear Analysis, vol. 3, no. 1, pp. 24-34, 2020.

[23] B. Hancioglu, D. Swigon, G.A. Clermont, A dynamical model of human immune response to influenza A virus infection, J Theor Biol., 246(1):70-86, 2007. 
[24] T.A. Henzinger. The theory of hybrid automata, Proceedings of the Eleventh Annual IEEE Symposium on Logic in Computer Science (LICS), pages 278-292, 1996.

[25] M. Kahraman, H. Öktem, G. W. Weber, M. Akhmet, Using Piecewise Linear Systems with Delay to Grab the Functional Dynamics in Biological Systems, HIBIT08 - International Symposium on Health Informatics and Bioinformatics, 2008.

[26] S. A. Kauffman, The Origins of Order: Self Organization and Selection in Evolution. Oxford University Press, New York, 1993.

[27] A. Khan, J.F. Gomez-Aguilar, T. Abdeljawad, H. Khan. Stability and numerical simulation of a fractional order plantnectarpollinator model. Alexandria Engineering Journal, 59(1):49-59, 2020.

[28] H. Khan., A. Khan, W. Chen, K. Shah. Stability analysis and a numerical scheme for fractional Klein-Gordon equations. Mathematical Methods in the Applied Sciences, 30;42(2):723-32, 2019.

[29] X. D. Koutsoukos, D. Riley. Computational Methods for Verification of Stochastic Hybrid Systems. IEEE Transactions on Systems, Man and Cybernetics, Part A, Volume 38, Issue 2, Page(s):385 - 396, 2008.

[30] V. A. Kuznetsov, I. A. Makalkin, M. A. Taylor, A. S. Perelson, Nonlinear dynamics of immunogenic tumors: Parameter estimation and global bifurcations analysis, Bull Math Biol., 56(2):295-321, 1994.

[31] J. Liu, A. R. Teel, Hybrid systems with memory: modeling and stability analysis via generalized solutions, IFAC World Congress, 2014.

[32] J. Lygeros, M. Prandini. Stochastic Hybrid Systems: A Powerful Framework for Complex, Large Scale Applications. European Journal of Control, 6: 583-594, 2010.

[33] N. Lynch, R. Segala, F. Vaandrager, H. B. Weinberg. Hybrid I/O automata,Lecture notes in computer science,pp. 496-510, 2000.

[34] A. Mantovani, M. Muzio, P. Ghezzi, C. Colotta, M. Introna, Regulation of inhibitory pathways of the interleukin-1 system, Ann. N. Y. Acad. Sci. 840, 338-351, 1998.

[35] H. Öktem, R. Pearson, K. Egiazarian, An Adjustable Aperiodic Model Class of Genomic Interactions Using Continuous Time Boolean Networks (Boolean Delay Equations), Chaos 13, 1167-1175, 2003.

[36] H. Öktem. Dynamic information handling in continuous time Boolean Network model of gene interactions, Nonlinear Analysis: Hybrid Systems, Volume 2, Issue 3, Pages 900-912, 2008.

[37] H. Öktem, A survey on piecewise-linear models of regulatory dynamical systems, Nonlinear Analysis, 63, 336-349,2005.

[38] M. Sainz-Trapága, C. Masoller, H. A. Braun, M. T. Huber, Influence of time-delayed feedback in the firing pattern of thermally sensitive neurons, Physical Review E, 70, 031904, 2004.

[39] A. J. van der Schaft, J. M. Schumacher, An Introduction to Hybrid Dynamical Systems, Lecture Notes in Control and Information Sciences, vol. 251 Springer, London, 2000.

[40] A. M. Selçuk, Hakan Öktem. An improved method for inference of piecewise linear systems by detecting jumps using derivative estimation. in: Nonlinear Analysis: Hybrid Systems, 3:3(277-287), 2009.

[41] P. Smolen, D. A. Baxter, and J. H. Byrne. Mathematical modeling of gene networks review, Neuron, vol. 26, no. 3, pp. $567-580,2000$.

[42] R. Thomas, Laws for the dynamics of regulatory networks, Int. J. Dev. Biol. 42, 479-485 (1998). 